\title{
Real-time global MHD simulation of the solar wind interaction with the earth's magnetosphere
}

\author{
H. Shimazu ${ }^{\mathrm{a}, \mathrm{b}, \mathrm{e}}$, K. Kitamura ${ }^{\mathrm{a}}$, T. Tanaka ${ }^{\mathrm{b}, \mathrm{e}, \mathrm{e}}$, S. Fujita ${ }^{\mathrm{d}, \mathrm{e}}$, M. S. Nakamura, ${ }^{\mathrm{a}, \mathrm{e}}$, and T. Obara, \\ ${ }^{a}$ National Institute of Information and Communications Technology, Koganei, Tokyo 184-8795 Japan \\ ${ }^{b}$ Space Environment Research Center, Kyushu University, Fukuoka, Japan \\ ${ }^{c}$ Department of Earth and Planetary Sciences, Kyushu University, Fukuoka, Japan \\ ${ }^{d}$ Meteorological College, Kashiwa, Chiba, Japan \\ ${ }^{e}$ CREST, JST \\ (Email: shimazu@nict.go.jp)
}

\begin{abstract}
We have developed a real-time global MHD (magnetohydrodynamics) simulation of the solar wind interaction with the earth's magnetosphere. By adopting the real-time solar wind parameters and interplanetary magnetic field (IMF) observed routinely by the ACE (Advanced Composition Explorer) spacecraft, responses of the magnetosphere are calculated with MHD code. The simulation is carried out routinely on the super computer system at National Institute of Information and Communications Technology (NICT), Japan. The visualized images of the magnetic field lines around the earth, pressure distribution on the meridian plane, and the conductivity of the polar ionosphere, can be referred to on the web site (http://www2.nict.go.jp/y/y223/simulation/realtime/).

The results show that various magnetospheric activities are almost reproduced qualitatively. They also give us information how geomagnetic disturbances develop in the magnetosphere in relation with the ionosphere. From the viewpoint of space weather, the real-time simulation helps us to understand the whole image in the current condition of the magnetosphere. To evaluate the simulation results, we compare the AE indices derived from the simulation and observations. The simulation and observation agree well for quiet days and isolated substorm cases in general.
\end{abstract}

\section{Introduction}

The interaction between the solar wind and the magnetosphere is a fundamental research topic of the space physics. Thanks to recent improvements in computer calculation speeds, larger-scale simulations have been performed. In the study of space plasma phenomena, simulation has played a significant role. Also, in recent years the behavior of space plasma near the earth has come to be referred to as "space weather," in consideration of its effect on human life. So we have a new opportunity to apply the fundamental research to the application of space weather.

Space weather includes a wide variety of phenomena. In this paper, we consider only the solar wind disturbances and the related magnetospheric and ionospheric phenomena. The solar wind disturbance can cause varieties of phenomena. For example, geomagnetic disturbances, radiation belt variation, and aurora activity.

Global simulation handles large systems that include regions of different characteristic temporal and spatial scales (solar wind, the magnetosphere, and the ionosphere). Since this type of simulation deals with large systems, the MHD simulation method is often adopted. The critical element of this research lies in the interaction between regions and the topology of magnetic field lines. These global MHD simulations have been used extensively for the study of the solar wind-magnetosphere interaction. Recently, a super computer system enables us to run the MHD simulation of the solar wind interaction with the earth's magnetosphere in real-time. We have developed a real-time global MHD simulation of the solar wind interaction with the earth's magnetosphere. In this paper, we describe the system in Section 2. Comparison with observations is described in Section 3.

(C) 2007. This manuscript version is made available under the Elsevier user license http://www.elsevier.com/open-access/userlicense/1.0/ 


\section{Real-time MHD Simulation System}

In the real-time MHD simulation system, we adopt the real-time solar wind parameters and IMF as input for the simulation (Den et al., 2006). Input parameters are the solar wind velocity, density, temperature and the $y$ and $z$ components of the IMF observed routinely by the ACE spacecraft in real time at 1-minute intervals. ACE, which was launched by NASA in 1997, is located at the L1 point (Zwickl et al., 1998). The distance between the $\mathrm{L} 1$ point and the earth is 1.5 million $\mathrm{km}$. It takes approximately 1 hour for the solar wind to propagate from the L1 point to the earth. In contrast, the radio waves propagate only in 5 seconds. Thus, we can get the information of the solar wind disturbances approximately in 1 hour advance. We are calculating the space weather conditions less than 1 hour before the solar wind arrives at the earth using the ACE data. One of the real-time ACE telemetry stations is located at NICT. Note that the real-time ACE data are preliminary unfortunately. Some corrections are necessary for scientific objectives.

The MHD code was developed by Tanaka (1994) to achieve highly accurate calculations through the application of the TVD method to the MHD simulation for systems with a potential magnetic field. He has advanced our understanding of the field-aligned current system (Tanaka, 1995) and of the structure and origin of the magnetospheric convection (Tanaka, 1999). The substorm mechanism was studied in relation with the convection (Tanaka, 2000).

In the real-time simulation, the inner boundary is located at 3 earth radii, where the ionosphere of the finite conductivity is placed. At the inner boundary, we solve equations for the conservation of the fieldaligned current. The conductivity is a function of the solar EUV flux, plasma pressure and density in the plasma sheet, and the field-aligned current. We adopt the modified spherical coordinates, and the grid numbers for this simulation are 44, 56, and 60 for the $\mathrm{r}$, theta, and phi direction, respectively.

We operate the simulation for 24 hours and 365 days in principle on the super computer system at NICT. The results are visualized, and the visualized images can be referred to the web site almost in real time (http://www2.nict.go.jp/y/y223/simulation/realtime/). We provide four kinds of panels (Fig. 1) in the web site. The upper left panel shows magnetic field lines around the earth. The upper right one shows the plasma pressure on the meridian plane. The center circle represents the earth. The bow shock and tail plasma sheet are observed. In the lower left one, the contour lines represent the electric potential, and the color code shows the electric conductivity of the polar ionosphere viewed from the north pole. The lower right one shows input parameters observed by ACE: solar wind velocity, density, and the $\mathrm{z}$ and $\mathrm{y}$ components of the IMF, respectively. The images are archived as movie format. We can refer to the past movies on the web site.

An example after an interplanetary shock hit the earth is shown in Fig. 2. Compare Fig. 2 with Fig.1 which is for the same day before the shock came. A pressure pulse propagates from the front side to the magnetotail. When the $\mathrm{z}$ component of the IMF becomes negative, the current sheet thinning and plasmoid ejection can be observed intermittently. At the same time of the plasmoid ejection, the conductivity of the polar ionosphere enhances on the night side. When the y component of the IMF is not oscillatory, clear convection cell structure is observed: round cell and crescent cell. It also shows that their shape depends on the direction of the y component of the IMF. The important point is that we can visualize the whole image of the magnetospheric conditions.

\section{Comparison with Observations}

It is important to link simulation results with observations. A satellite observes at only one point, and it is difficult to get the whole image in general even if we use many satellites. In contrast, the global simulation can visualize the whole image and conditions in the magnetosphere. The real-time simulation data and the observation data can be complementary.

Simulation is based on the physics law, but we have made some approximation and include numerical errors. Thus, how the simulation can explain the real observation is a very important question. Through comparison with observation data, simulation results can be verified. Moreover, simulations in which conditions can be set freely represent indispensable tools in the understanding of the essential elements of observational results. 
To compare with the observation, it is important to compare the same physical parameters. In this paper, we use the AE, AU, AL and $\mathbf{A O}$ indices for this comparison. The indices are derived from $\mathbf{1 0}$ geomagnetic observatories near the aurora oval almost separated equidistantly in the longitude. The indices are better for the comparison with the simulation than data from satellites, because the indices are derived by integrating (overlay) over the wide area. Locality can be exaggerated in one-point observation.

The virtual $\mathrm{AE}, \mathrm{AU}, \mathrm{AL}$ and $\mathrm{AO}$ indices are derived in the simulation from the $\mathrm{H}$ (horizontal) component at all grid points located at latitudes between 60 and 70 degrees, which is different from the derivation of the real indices. There are some differences between the virtual indices derived from the all grid-point data and those derived from 10 grid points near the observatories. For example, the virtual indices derived from the all grid-point data are sometimes overestimated compared with the virtual indices derived from the 10 grid points, and the timing of the variations is not always coincident. However, the difference between them is small compared with the difference from the real indices. Here, we use the virtual indices derived from the all grid-point data.

Fig. 3 shows the AE, AU, AL and AO indices obtained in this simulation and in the observation for oneday period on September 3, 2005. We use one-minute value for both simulation and observation. The virtual indices are based on the real-time solar wind data which are obtained by ACE. Thus, we must consider the propagation time for the solar wind from ACE to the earth. Since it depends on the structure in the solar wind and the IMF as well as the solar wind velocity, it is difficult to estimate the propagation time only from the solar wind velocity. Here, we calculate the cross-correlation coefficients of the real indices to the virtual indices in each day. Time series of the virtual indices are shifted from 0 to 120 minutes with the step of one minute. The propagation time is defined as the shifted time at which the correlation coefficient shows the maximum value. The propagation times are generally different in each day. On the day shown in Fig. 3, the propagation time is $\mathbf{7 3}$ minutes.

Fig. 3 indicates that the general trends of the simulation and observation agree well. The maximum correlation coefficients of the $\mathrm{AE}, \mathrm{AU}, \mathrm{AL}$ and $\mathrm{AO}$ indices are $\mathbf{0 . 8 2 5}, \mathbf{0 . 6 6 5 , 0 . 8 0 1}$ and $\mathbf{- 0 . 3 8 8}$, respectively. The activity level and timing are almost reproduced except for the $\mathbf{A O}$ index, if we do not consider variations shorter than 10 minutes. In this case, the IMF had a weak southward component for almost all day long and isolated substorms occurred.

Here, we consider the AE index because it is the most popular in the four indices for monitoring auroral activities. Fig. 4 shows the statistics of the maximum correlation coefficients between the simulation and observation for each day. Valid data of 247 days from August 2005 to June 2006 are used. The correlation coefficients show quite good with more than 0.5 in 158 days of the total 247 days $(64 \%)$. Analysis of these events shows that both simulation and observation agree well for quiet days and isolated substorm cases in general. It does not show good correlations when the geomagnetic disturbance is severe. We found that the mesh number and resolution adopted in the simulation is a critical parameter for poor correlation cases. Details about the agreement and disagreement will be discussed in another paper.

\section{Summary}

The real-time MHD simulation of the interaction between the solar wind and the magnetosphere has been developed. The results show that various magnetospheric activities are almost reproduced. It is important to link simulation results with observations. We compare the $\mathrm{AE}$ indices between the simulation and the observation. In general, correlation is quite good for quiet days and isolated substorm cases. By analyzing the data and comparing them with observations, we are continuously modifying our model. We believe that the real-time simulation is being improved continuously and approaching a more realistic magnetosphere.

\section{Acknowledgements}

This work was supported by NICT project y223103 and CREST, JST. 


\section{References}

Den, M., T. Tanaka, S. Fujita, T. Obara, H. Shimazu, H. Amo, Y. Hayashi, E. Nakano, Y. Seo, K. Suehiro, H. Takahara, and T. Takei, Real-time earth's magnetosphere simulator with 3-dimensional MHD code, Space Weather, 4, doi: 10.1029/2004SW000100, 2006.

Tanaka, T., Finite volume TVD scheme on an unstructured grid system for three-dimensional MHD simulation of inhomogeneous systems including strong background potential fields, J. Comput. Phys., 111, 381-389, 1994.

Tanaka, T., Generation mechanisms for magnetosphere-ionosphere current systems deduced from a threedimensional MHD simulation of the solar wind-magnetosphere-ionosphere coupling processes, $\mathrm{J}$. Geophys. Res., 100, 12057-120734, 1995.

Tanaka, T., Configuration of the magnetosphere-ionosphere convection system under northward IMF conditions with nonzero IMF By, J. Geophys. Res., 104, 14683-14690, 1999.

Tanaka, T., The state transition model of the substorm onset, J. Geophys. Res., 105, 21081-21096, 2000.

Zwickl, R. D., K. A. Doggett, S. Sahm, W. P. Barrett, R. N. Grubb, T. R. Detman, V. J. Raben, C. W. Smith, P. Riley, R. E. Gold, R. A. Mewaldt, and T. Maruyama, The NOAA Real-Time Solar-Wind (RTSW) system using ACE data, Space Sci. Rev., 86, 633-648, 1998. 


\section{Figure Captions}

Fig. 1: An example of panels shown in the web site. (upper left panel) Magnetic field lines connected to the earth. The blue ones are connected to the northern hemisphere, and the red ones are connected to the southern hemisphere. Some are blue and others are red for field lines whose ends are connected to both hemispheres. (upper right panel) Plasma pressure on the meridian plane. The center circle represents the earth. (lower left panel) The contour lines represent the electric potential and the color code shows the electric conductivity of the polar ionosphere viewed from the north pole. (lower right panel) Input parameters observed by the ACE spacecraft: solar wind velocity, density, and the $\mathrm{z}$ and $\mathrm{y}$ components of the IMF, respectively.

Fig. 2: The format is as the same as Fig. 1, but the panels are for disturbed conditions after an interplanetary shock hit the earth.

Fig. 3: $\mathrm{AE}, \mathrm{AU}, \mathrm{AL}$ and $\mathrm{AO}$ indices obtained in this simulation and in the observation for one day period on September 3, 2005. Solid lines represent the simulation results and dotted line represents the observation.

Fig. 4: Histogram of the maximum correlation coefficients between the simulation and observation for each day. 


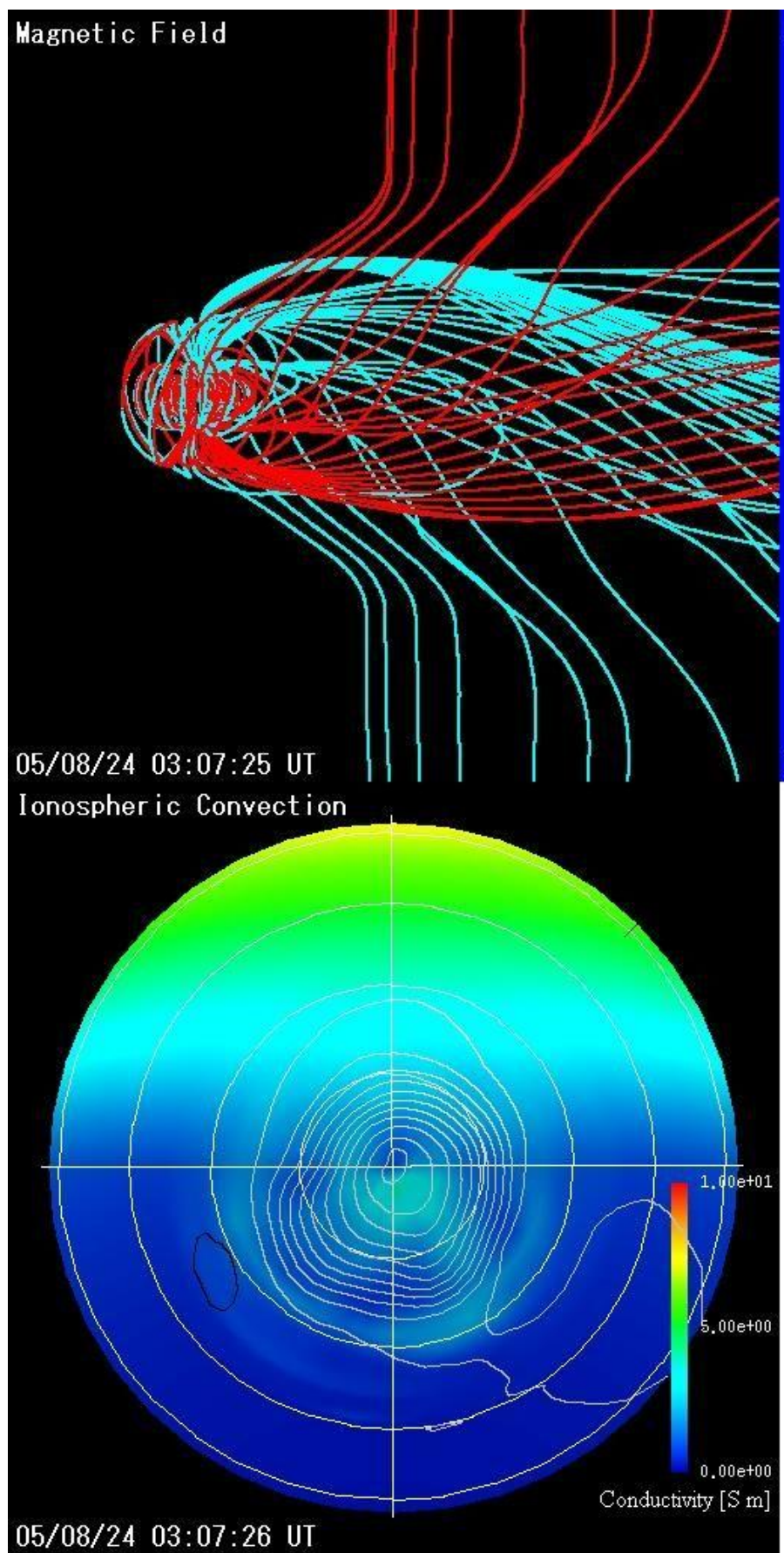

Pressure

Pressure
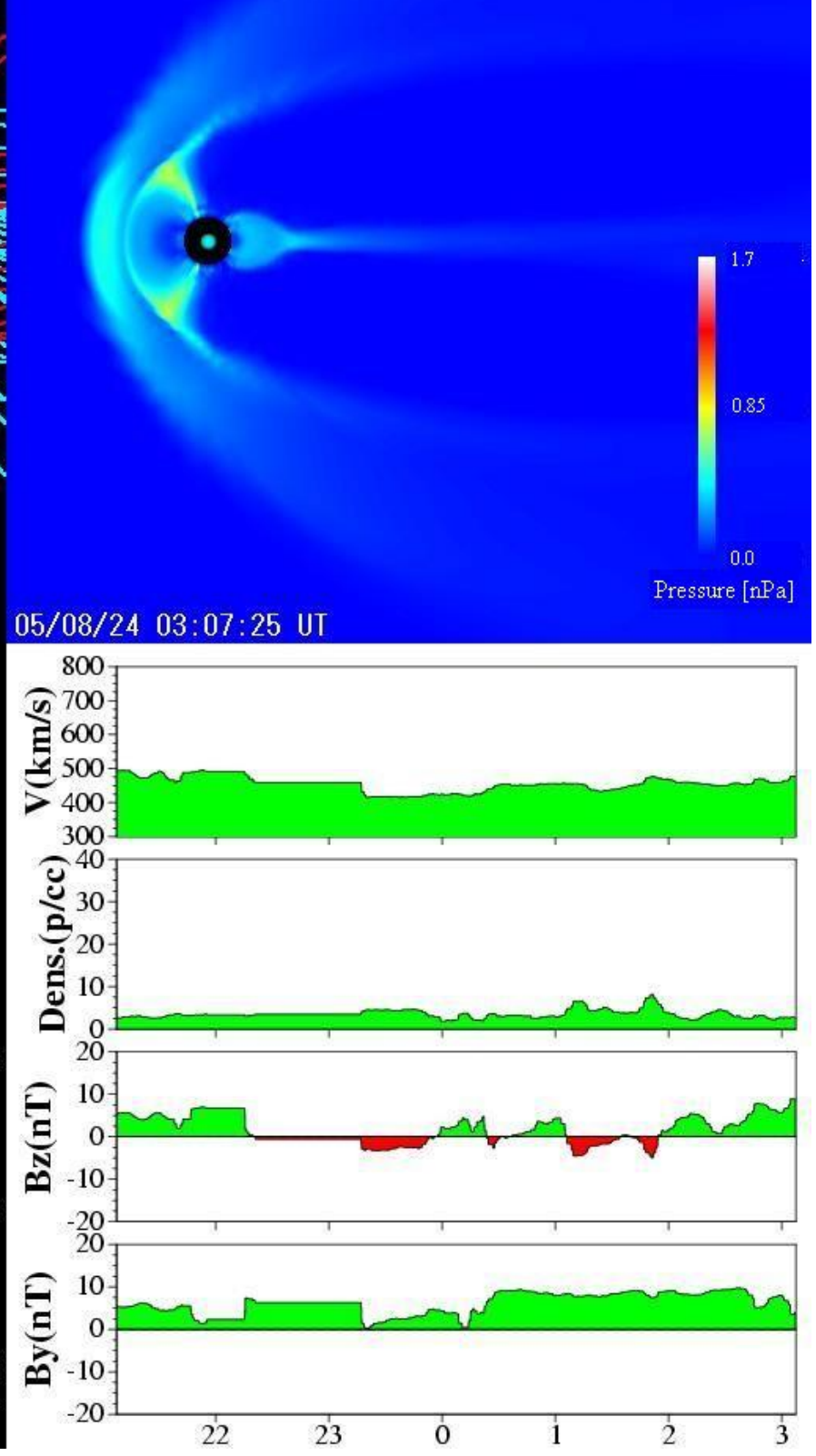

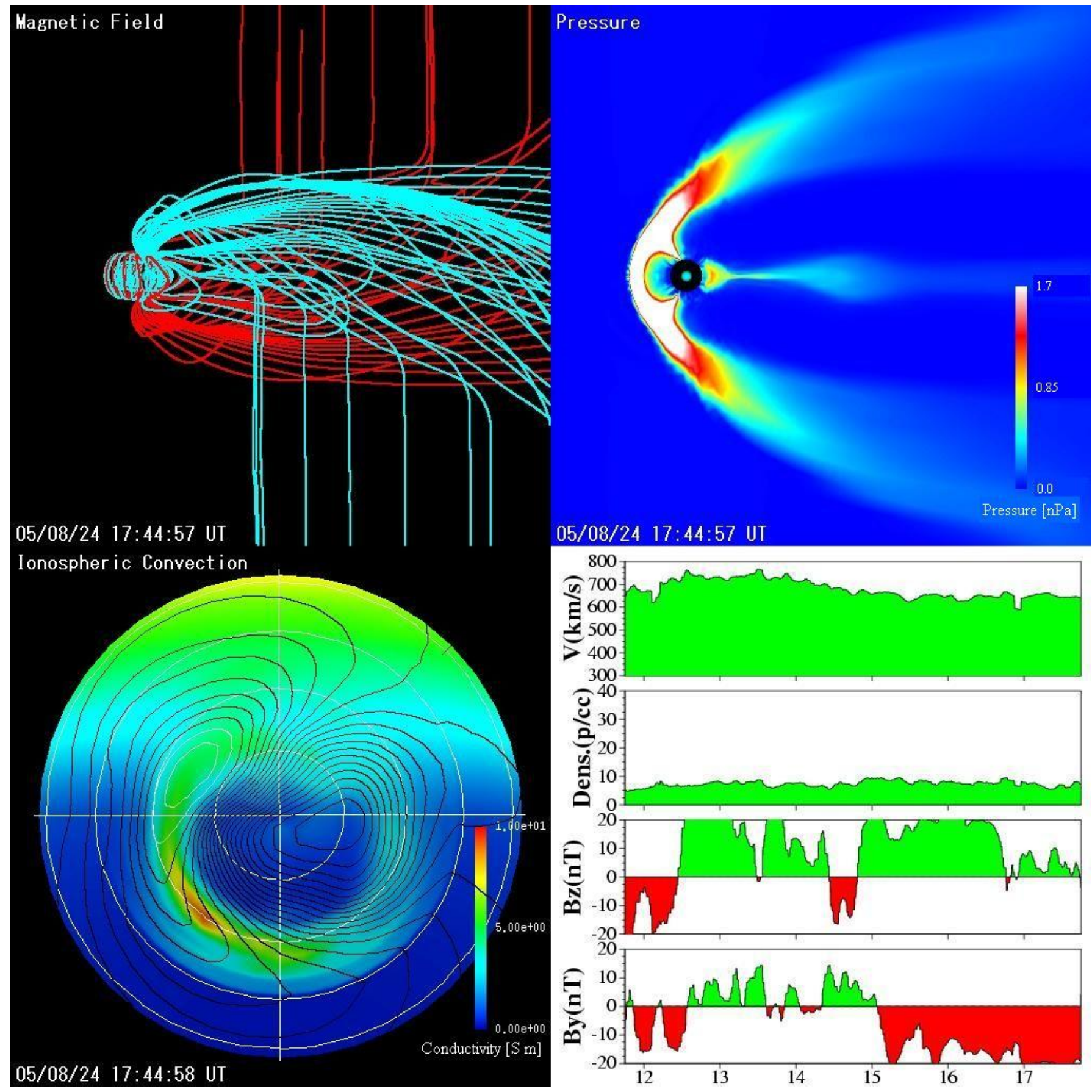

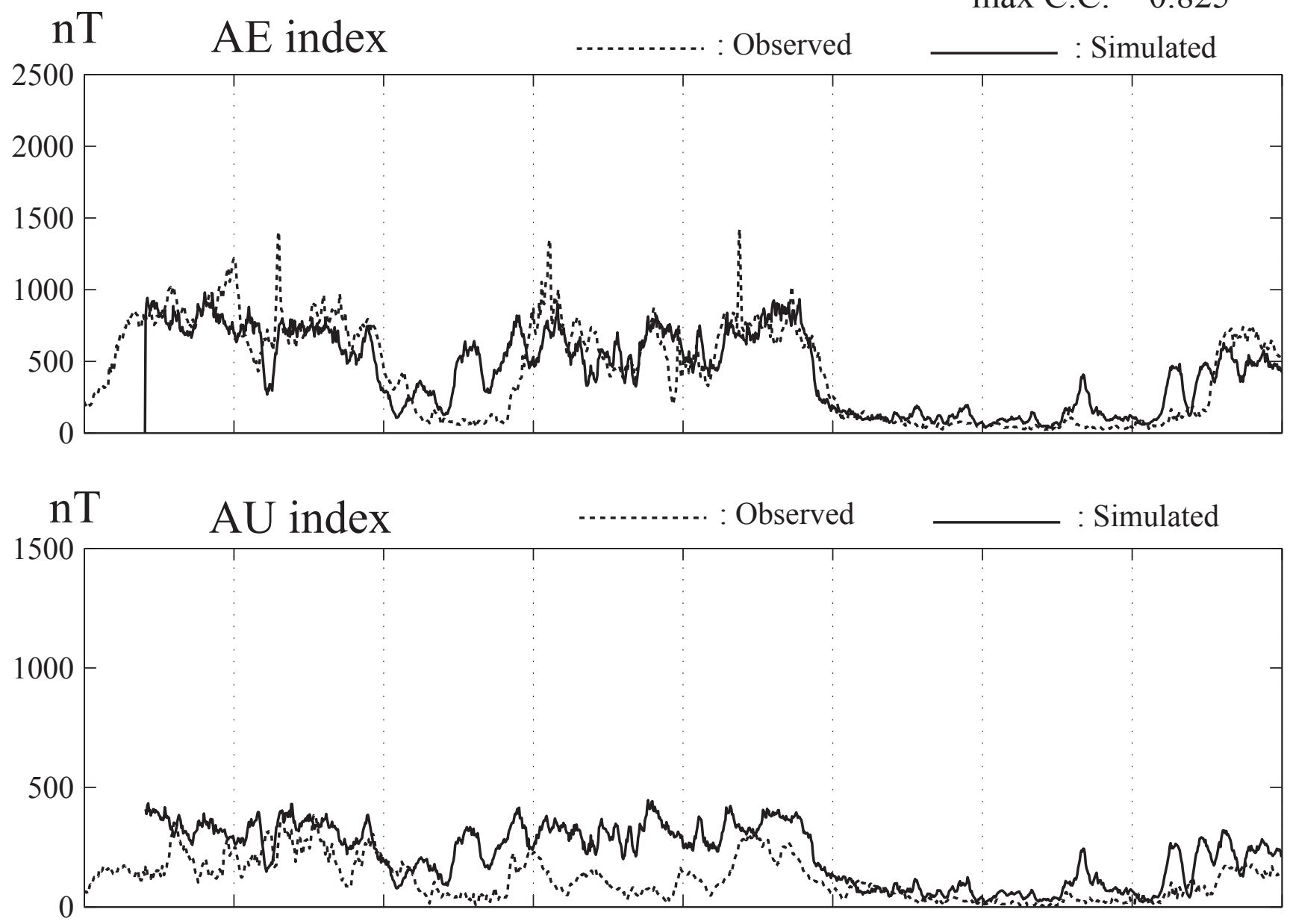

nT AL index

: Observed : Simulated
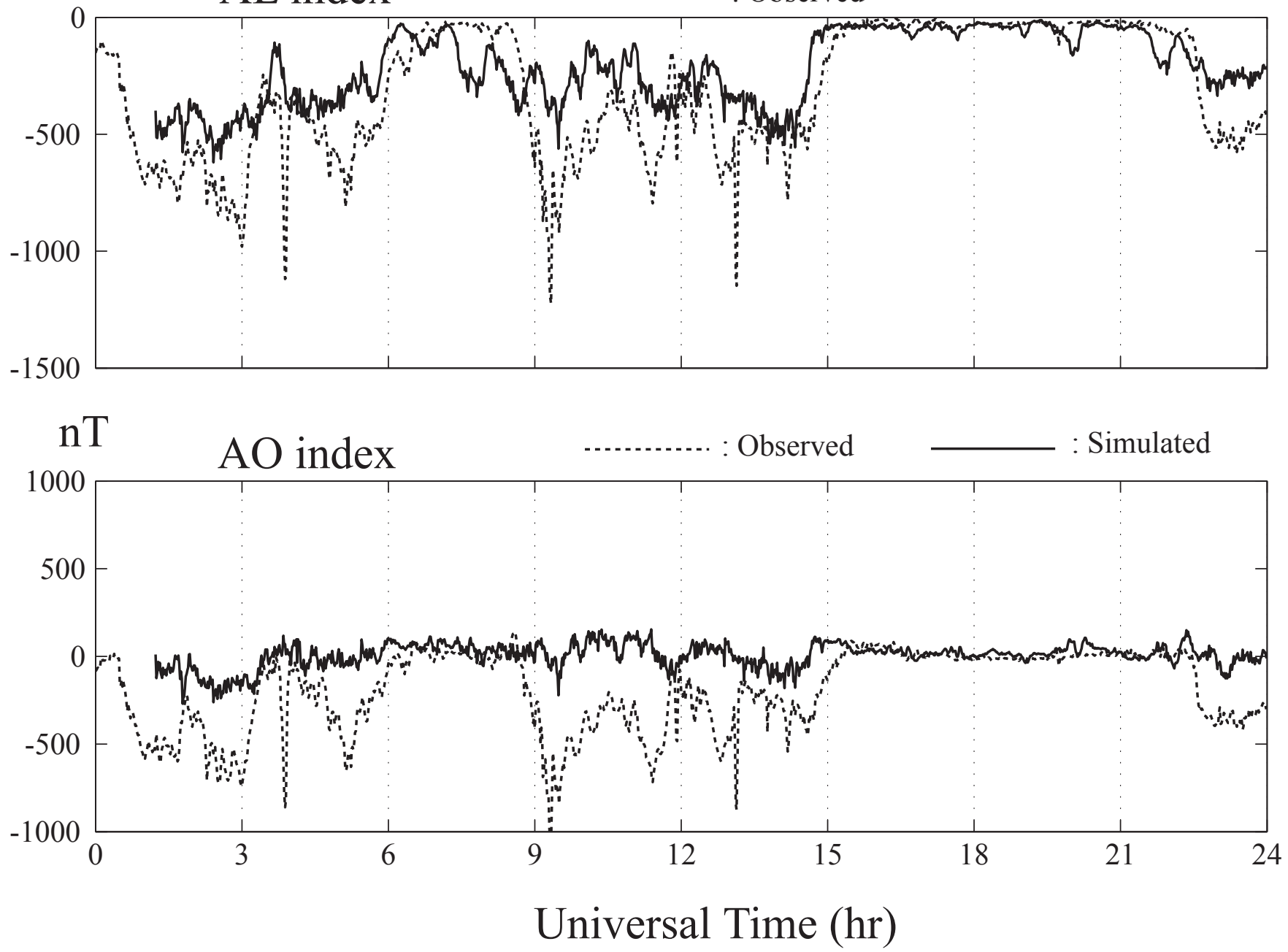


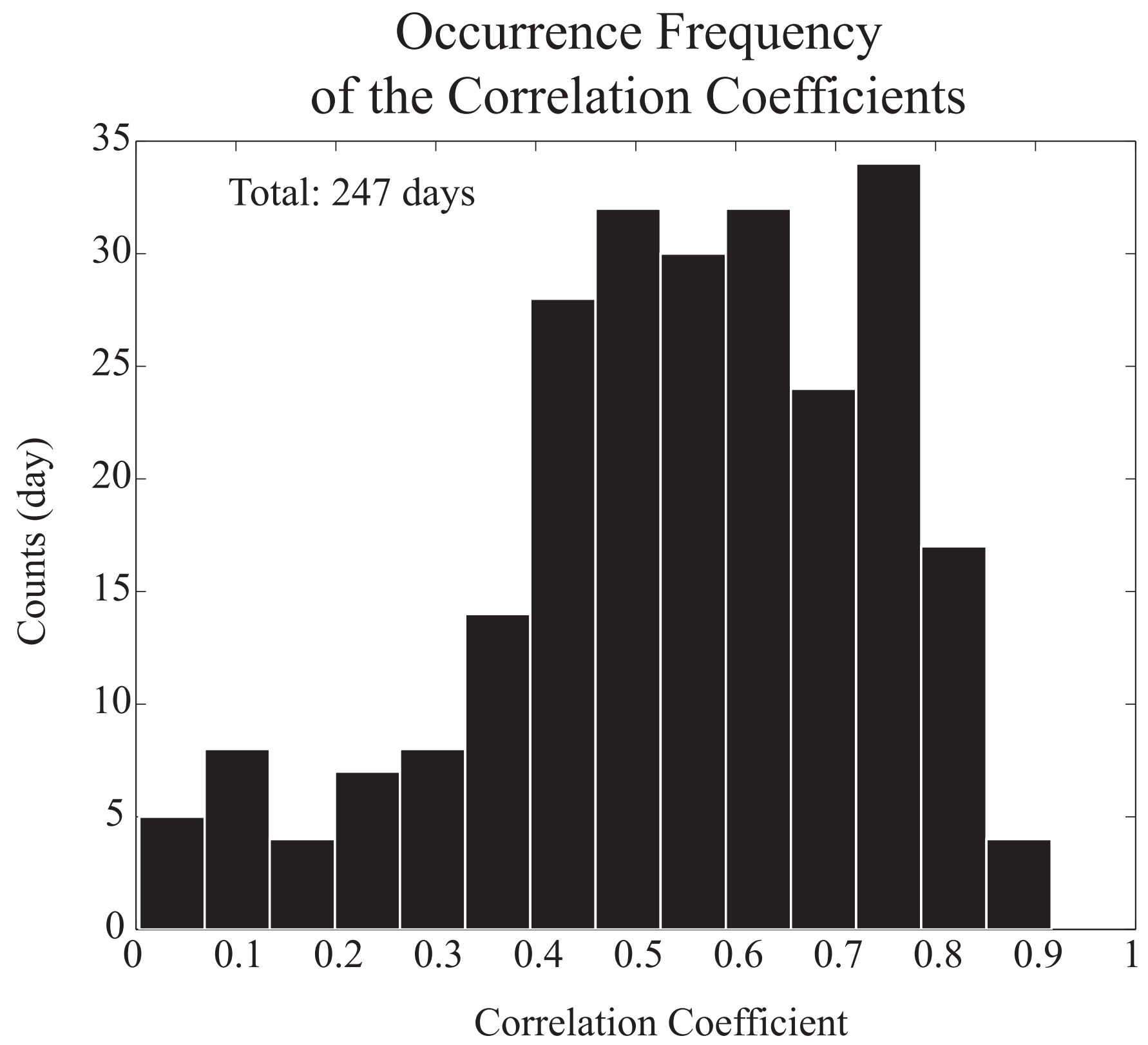

